\title{
Daniele Lorenzini et Ariane Revel (dir.), Le Travail de la littérature. Usages du littéraire en philosophie
}

Rennes, Presses universitaires de Rennes, 2012, 256 p.

\author{
Sathya Rao \\ Université de l'Alberta
}

Dirigé par Daniele Lorenzini et Ariane Revel, Le Travail de la littérature. Usages du littéraire en philosophie regroupe les versions remaniées de présentations données dans le cadre d'un colloque international organisé en mai 2011 par l'équipe «Lettres, idées, savoirs » de l'université Paris-Est Créteil et le Centre international d'étude de la philosophie française contemporaine de l'École normale supérieure de la rue d'Ulm. Cet ouvrage, qui compte des contributions de penseurs de renom au nombre desquels Pierre Macherey, Frédéric Worms, 
Sandra Laugier et Arnold I. Davidson, est divisé en trois parties intitulées "Penser avec la littérature», "La philosophie à l'épreuve du texte» et «La littérature, expérimentation et attention morale ». L'introduction de Daniele Lorenzini et d'Ariane Revel a le mérite de poser clairement les termes de la problématique qui guide Le Travail de la littérature. Il s'agit non pas de soumettre la littérature à l'ordre de la philosophie, mais de comprendre sous quelles modalités celle-ci «travaille » celle-là et se trouve travaillée en retour. On le sait, le rapport entre philosophie et littérature prend place au sein d'une histoire aussi longue que complexe dont Philippe Sabot a tenté de faire la généalogie dans Philosophie et littérature. Approches et enjeux d'une question. Rappelant brièvement cette histoire, Daniele Lorenzini et Ariane Revel s'efforcent, à leur tour, de la problématiser en interrogeant l'arbitraire de la distinction des genres, en identifiant des «fonctions» littéraires du discours philosophique ou encore en questionnant la nature profondément philosophique de l'expérience littéraire. Dans le sillage de la typologie de Sabot, elles prennent le parti de poser une «absence d'extériorité » entre philosophie et littérature dont on pourra regretter qu'elle n'ait pas fait l'objet d'une amorce plus substantielle de théorisation, de même que le concept de «travail ».

La première section de l'ouvrage, qui porte le titre "Penser la littérature », s'attache à identifier la nature exacte de l'expérience littéraire au moyen d'outils que l'on devine philosophiques. Mettant en parallèle la lecture que fait André Breton des Chants de Maldoror et les interprétations que donnent à la fois Bakhtine et Léo Spitzer de Gargantua, Pierre Macherey met en évidence la fonction critique de la littérature, laquelle rappelle à la philosophie - ou tout au moins à une 
certaine philosophie demeurant sous la tutelle de la métaphysique - de ne pas prendre sa quête de la vérité trop au sérieux. Reposant sur une conception quelque peu classique de la philosophie, l'argumentaire de Pierre Macherey devrait certainement être révisé s'agissant de philosophies antimétaphysiques comme celles de Nietzsche et de Derrida. En ce qui concerne Barbara Carnevali, elle met à profit le cadre interprétatif de l'œuvre proustienne élaboré par Vincent Descombes (qui s'inspire, pour sa part, de René Girard) pour tirer une "philosophie sociale » à l'œuvre chez Proust. La thèse principale de l'auteure est que l'écrivain français aurait découvert une "esthétique sociale» envisageant la forme sensible comme le «médium esthétique, le mode d'expression et de constitution des rapports sociaux, et donc aussi de leur reproduction et de leur connaissance» (p.42). Si elle est incontestablement originale, la lecture de Barbara Carnevali qui emprunte tout autant à Bourdieu qu'à Danto - ne marque pas clairement la distinction entre philosophie et sociologie; ce qui, compte tenu de la thématique de l'ouvrage, pourrait s'avérer problématique. La contribution de Frédéric Worms, intitulée «De l'autofiction à l'œuvre-témoignage. Littérature et philosophie dans le moment vivant », quant à elle, s'attache à mettre en évidence la nouvelle actualité des rapports entre vie et écriture. Ce moment contemporain du vivant - dont l'auteur préfère prendre acte plutôt que de retracer la généalogie - se signale par l'émergence de deux genres littéraires antinomiques ou extrêmes, à savoir l'œuvre-témoignage et l'autofiction. Tandis que le premier prévient la confusion entre vie et écriture au terme d'un mouvement de double sublimation, la seconde aurait plutôt tendance à en brouiller les frontières. On pourra regretter toutefois que le cadre d'analyse proposé par Frédéric 
Worms - dont la pertinence est, au demeurant, évidente fasse un usage restreint de la philosophie. Comme il le rappelle en conclusion, le concept de vivant connaît de multiples élaborations philosophiques contemporaines de Foucault, Giorgio Agamben en passant par Michel Henry, dont il faudrait également saisir l'actualité des manifestations littéraires. Dans la contribution qu'il consacre à Michel Foucault, Jérémy Romero s'attache à montrer que, pour le philosophe français, la littérature s'apparente à une forme d'anéantissement en déliant le lien naturel entre les mots et les choses. Au fil d'une étude comparée sur l'idée de la littérature que se font Nietzche, Hegel et Heidegger, le critique établit à la fois l'originalité de la conception qui est celle de Foucault et la centralité de la question littéraire au sein de l'édifice philosophique foucaldien (en particulier, dans son rapport avec les concepts d'« expérience » et de « contestation »). Dans « Prose ou monde ou ordre du discours? La littérature, un enjeu politique », Judith Revel s'intéresse à deux cas où la littérature possède une fonction de «révélateur » pour la philosophie, à savoir ceux de Merleau-Ponty et de Michel Foucault. Dans le sillage de la contribution de Jérémy Romero, elle dégage deux moments antinomiques mais complémentaires du traitement foucaldien de la question du langage, à savoir celui de l'archéologie des discours et celui, moins connu, de l'ésotérisme structural qui porte sur l'examen d'interventions littéraires déviantes comme celles de Raymond Roussel, Georges Bataille et Maurice Blanchot. En définitive, la question de la littérature porte chez Foucault la possibilité d'une sortie (politique) hors de l'orthodoxie du discours et donc d'une forme d'invention au sein de ce dernier. C'est justement le concept de " chiasme » utilisé par Foucault pour décrire la dialectique précédente qui 
constitue le trait d'union avec Merleau-Ponty. Chez ce dernier, la littérature (et la peinture) constitue non seulement un révélateur, mais aussi l'outil d'un renouvellement de l'histoire et du langage : «La leçon de l'écrivain, c'est que l'on n'est jamais aussi puissant que lorsqu'on plie la panoplie des mots partagés et des significations disponibles à la puissance d'inauguration de l'écriture [...]. » (p. 99)

La seconde section de l'ouvrage s'intéresse à la prise en compte d'œuvres littéraires dans l'étude philosophique de la littérature. L'article de Martin Rueff s'intéresse aux modalités de l'inscription de l'éthique dans la littérature dans le cadre d'une poétique du discours moral d'inspiration wittgensteinienne. Il identifie ainsi plusieurs catégories métaéthiques permettant d'appréhender l'inscription de la moralité dans le discours (littéraire). En ce qui concerne la contribution de Vincent Delecroix, elle envisage la philosophie comme un genre littéraire dans un triple sens critique, méthodologique et prescriptif. La littérature, dont il faudrait se garder d'hypostasier le régime de vérité, permettrait à la philosophie de se former à l'école du singulier. Pratiquer la philosophie comme littérature, c'est en définitive reconnaître que c'est la fiction qui fait le lit de la prétention de vérité de la philosophie, que celle-ci n'est en fin de compte qu'un " produit de la vérité » plutôt que la vérité elle-même. Pour sa part, Philippe Sabot revient sur un usage dominant du littéraire en philosophie, à savoir celui consistant à lui accorder une vocation cognitive, en particulier dans le champ de la «vie morale ». Pour ce faire, il met à l'épreuve l'analyse que livre Bouveresse sur le «roman expérimental» zolien dans $L a$ Connaissance de l'écrivain. Pour Philippe Sabot, l'inconvénient de la position de Bouveresse est qu'elle prête indistinctement à 
toutes les œuvres littéraires un égal « esprit éthique » au risque de restreindre les vertus de la littérature au seul domaine de l'éducation à la vie morale. À l'esprit éthique de Bouveresse, Philippe Sabot oppose une éthique de l'écriture inspirée par Foucault, qu'il ne présente malheureusement que très brièvement dans une note en bas de page à la fin de l'article. Dans «Stylistique de l'existence entre philosophie et littérature », Marielle Macé s'intéresse aux façons dont philosophie et littérature peuvent permettre d'appréhender les formes de vie dans le cadre d'un projet plus large de «stylistique de l'existence ». À cet égard, l'œuvre de Balzac est remarquable dans la mesure où elle prend le «style en charge et en responsabilité » (p.152). Marielle Macé trouve dans l'«attitude esthétique» conceptualisée par Foucault l'orientation du projet littéraire d'un Balzac tandis qu'il parvient, à force d'observation, à élever la vie au statut de configuration stylistique au-delà de toute discrimination et distinction sociales.

La troisième et dernière partie de l'ouvrage, intitulée "Littérature, expérimentation et attention morale», qui se présente davantage comme une suite d'études de cas, s'ouvre avec une contribution de Sandra Laugier qui, dans le sillage de Cora Diamond, envisage l'éthique moins comme une théorie normative que comme un travail authentiquement exploratoire tourné vers l'ordinaire. Mise à profit dans le cadre de cette exploration, la littérature ne fait toutefois pas l'objet d'une mise en question à proprement parler (par exemple, à travers la catégorie littéraire de l'ordinaire ou du quotidien). Pour sa part, Solange Clavel s'attache à illustrer la pertinence de la littérature pour comprendre le concept de frontières tel qu'il est élaboré par la philosophie politique. Plutôt que d'imposer une doctrine 
définitive des frontières, les expériences fictionnelles qu'en proposent Jünger, Buzzati et Coetzee montrent la diversité des organisations imaginaires du monde. L'article d'Emmanuel Halais s'attache, lui, à mettre en évidence la portée philosophique de De Profundis d'Oscar Wilde à travers l'examen thématique de l'œuvre. Dans «Circulation, résistance, perméabilité. Cavell et la littérature écologique américaine», Marie Satya McDonough explore la proximité entre l'œuvre de Stanley Cavell et deux textes étasuniens de la littérature environnementale des années 1960 à travers les concepts de communauté et de conversation. Atypique, la contribution d'Arnold I. Davidson, qui clôture l'ouvrage, examine le modèle d'improvisation du saxophoniste Sony Rollins dans la mesure où il fait le lien entre le perfectionnisme moral de Stanley Cavell et la pratique des exercices spirituels au sens de Pierre Hadot.

Regroupant plusieurs contributions d'excellente qualité, Le Travail de la littérature propose une réflexion à la fois diversifiée et contemporaine sur les rapports entre littérature et philosophie. Un des nombreux mérites de l'ouvrage est d'inclure dans la conversation la philosophie anglo-saxonne (Wittgenstein, Cavell, etc.) tout en s'efforçant de préserver la littérature de la menace de son instrumentalisation philosophique; ce qui renforcera l'intérêt du livre aux yeux des littéraires. Certaines contributions comme celles de Pierre Macherey et de Marie Satya McDonough pourront apparaître quelque peu décevantes au regard de la qualité générale de l'ouvrage. Si elle est dans l'ensemble cohérente et bien justifiée, l'organisation de l'ouvrage n'est pas sans soulever quelques interrogations. On pourra ainsi se demander pourquoi la contribution de Barbara Carnaveli et de Marielle Macé, qui proposent des approches similaires, n’ont pas été regroupées. 